\title{
Trajectory Tracking for the Bebop Parrot quadrotor using Takagi-Sugeno fuzzy models
}

\author{
Rayza Araujo* Flávio A. Faria ${ }^{* *}$ Leandro J. Elias ${ }^{* * *}$ \\ Vilma A. Oliveira* \\ * Depto Engenharia Elétrica e Computação, Universidade de São \\ Paulo, São Carlos, SP (e-mail: rayza.araujo@usp.br,voliveira@usp.br) \\ ** Depto de Físico Química, Instituto de Química, São Paulo State \\ University (UNESP), Araraquara,SP (e-mail: flavio.faria@unesp.br) \\ *** Depto de Matemática e Educação, Instituto Federal de Educação, \\ Ciência e Tecnologia de São Paulo, Araraquara, SP (e-mail: \\ leandro.elias@ifsp.edu.br)
}

\begin{abstract}
In this work, we propose a control strategy to solve the 3D positioning of a commercial quadrotor. A parallel distributed compensation (PDC) fuzzy controller is implemented using the tracking error equations. The results are developed for a Lyapunov fuzzy function and are solved in terms of linear matrix inequalities (LMI)s. The experimental results show the efficiency of the fuzzy controller to perform trajectory tracking.
\end{abstract}

Keywords: Lyapunov fuzzy function, unmaned aerial vehicles, error dynamics.

\section{INTRODUCTION}

Unmanned Aerial Vehicles (UAVs) have been increasingly gaining popularity in robotics for their vast range of possible applications, varying from leisure to military. Among the UAVs, the quadrotor deserves special attention for its habilities to hover and to take off and land vertically. This makes quadrotors more flexible, given that they do not require a runway.

A number of nonlinear and adaptive control techniques have been applied to quadrotors due to their inherent parametric uncertainties and coupled nonlinearities (Mo and Farid, 2019). Santana et al. (2014) uses feedback linearization techniques to control the trajectory of a Parrot AR.Drone. Unmodeled dynamics are a concern when using this type of controller, as it can even destabilize the system. Tang and Li (2015) combined Linear Quadratic Regulator (LQR) and sliding mode techniques to obtain a tracking controller, however, the simulation therein shows that the closed loop was too slow to follow a more complex trajectory such as a circle.

In the literature, fuzzy techniques have been considered to control quadrotors, including experimental results (Ponce et al., 2015; Sampath et al., 2014). In the former, the authors use a fuzzy logic type 2 controller with an Adaptive Neuro-Fuzzy Inference System to optimize the number of membership function of a quadrotor position. In the latter, the authors use a fuzzy logic type Mandani controller with 121 rules based on experimental data to design a fuzzy controller for a quadrotor attitude control.

The Takagi Sugeno (TS) fuzzy modelling can represent a large class of nonlinear systems by means of a sum of averaged linear models (Tanaka and Wang, 2001), which uses the sector nonlinearities approach. In this approach, a minimum number of membership functions is used to describe the nonlinear system exactly. A characteristic of the TS fuzzy modelling is that stability can be analyzed using linear matrix inequalities (LMIs) in a Lyapunov formulation, which can be efficiently solved by convex programming techniques. A fuzzy dependent Lyapunov function is less conservative than a quadratic one and because of that has gained a lot of attention (Faria et al., 2013).

Most applications of Takagi Sugeno control found in the literature are simulations. In Koslosky et al. (2017) a fuzzy control system to control the movement of a hexacopter was developed. The controller was implemented using ROS Indigo and the results simulated on V-REP (robotics simulator). Grijalva et al. (2017) obtained simulation results for discrete time control of a quadrotor using TS fuzzy modeling combined with integral action to ensure null steady state error. The authors demonstrated the tracking performance using piecewise steps rather than the usual tracking references. To our knowledge, experimental results using Takagi Sugeno fuzzy modelling in tracking applications are lacking.

In this work, we implemented a control system on a off-theshelf quadrotor aiming to follow a given trajectory. The system was modeled using the Takagi-Sugeno approach and a PDC fuzzy controller was designed. The control loop was implemented using the Robot Operating System (ROS) and experimental data were obtained.

This paper is organized as follows. In the next section, we describe the configuration of the control system. Section 3 follows with the details of the quadrotor model used and the derivation of the tracking error model. In Section 4, we derive the Takagi Sugeno fuzzy model and design the PDC controller. In Section 5 we give details of the control 
system implementation. In Section 6 we show experimental results. Finally, the concluding remarks appear in Section 7.

\section{CONTROL SYSTEM OVERVIEW}

The control objective of the quadrotor is to track a given trajectory. We approach this tracking problem by designing a stabilizing controller for the error system. This approach results in a much simpler problem, as the controller's task is merely to take the error system to the origin. For this, any stabilizing controller design technique can be used.

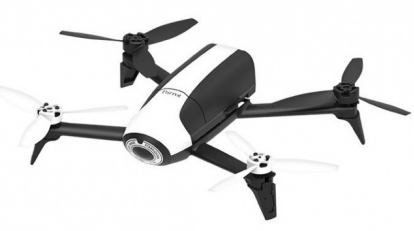

Figure 1. Parrot BEBOP 2.0: Parrot $\mathbb{R}$

The quadrotor to be controlled is a BEBOP Parrot 2.0 (see Figure 1), chosen for its flight stabilization, available SDK and the possibility of ROS integration. The disadvantage of using an off-the-shelf platform is that the access to its inner control loops for hovering and flight stabilization is not given. On the other hand, having these inner control loops available simplifies the dynamics which, for a regular quadrotor, are very complicated and nonlinear (Bresciani, 2008). Simpler dynamics often result in simpler control design.

To account for the nonlinearities, a state feedback fuzzy controller in a PDC form is used. Figure 2 illustrates the implemented control system. The trajectory generator calculates the actual desired position and orientation based on a predefined trajectory and feeds this information to the designed controller. A VICON motion capture system is used to measure the quadrotor's position and orientation. Since the state is formed by the tracking position and velocity error, a Kalman filter estimates the quadrotor's velocity using the available measurements. Then, the tracking error is calculated and its corresponding control law is sent to the Parrot Bebop through the network, closing the control loop.

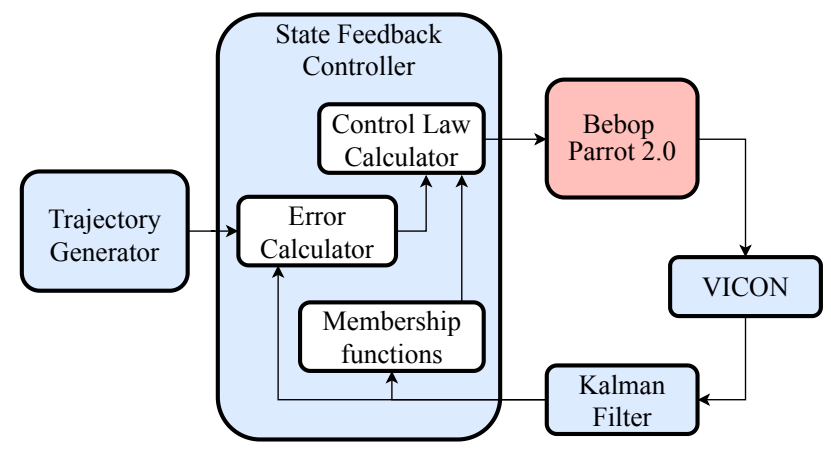

Figure 2. Control system block diagram showing the controller loop.

\section{PARROT BEBOP MODEL}

Considering the aforementioned internal control loops, the quadrotor's response to the input command vector $u=\left[\begin{array}{llll}u_{\nu_{x}} & u_{\nu_{y}} & u_{\dot{z}} & u_{\dot{\psi}}\end{array}\right]^{T}$ can be modeled as the following linear system (Santana et al., 2014)

$$
\ddot{q}_{b}=\Gamma_{A} \dot{q}_{b}+\Gamma_{B} u
$$

where $q_{b}=\left[\begin{array}{llll}x_{b} & y_{b} & z_{b} & \psi_{b}\end{array}\right]^{T}$ is the pose vector with respect to the body frame. The model matrices are given by

$$
\Gamma_{A}=-\left[\begin{array}{cccc}
\gamma_{2} & & & \\
& \gamma_{4} & & \\
& & \gamma_{6} & \\
& & & \gamma_{8}
\end{array}\right], \Gamma_{B}=\left[\begin{array}{llll}
\gamma_{1} & & & \\
& \gamma_{3} & & \\
& & \gamma_{5} & \\
& & & \gamma_{7}
\end{array}\right]
$$

where $\gamma_{i}$ are proportionality constants to be defined experimentally and vary among platforms. Rotating this model to a global reference frame and defining a new state vector as $\zeta=\left[\begin{array}{ll}\dot{q} & q\end{array}\right]^{T}=\left[\begin{array}{llllllll}\dot{x} & \dot{y} & \dot{z} & \dot{\psi} & x & y & z & \psi\end{array}\right]^{T}$, we obtain the following state space model:

$$
\dot{\zeta}=\left[\begin{array}{cc}
-N R^{T} & 0 \\
I & 0
\end{array}\right] \zeta+\left[\begin{array}{c}
M \\
0
\end{array}\right] u
$$

where the model matrices are given by

$$
\begin{aligned}
M & =\left[\begin{array}{cccc}
\gamma_{1} \cos \psi & -\gamma_{3} \sin \psi & 0 & 0 \\
\gamma_{1} \sin \psi & \gamma_{3} \cos \psi & 0 & 0 \\
0 & 0 & \gamma_{5} & 0 \\
0 & 0 & 0 & \gamma_{7}
\end{array}\right] \\
N & =\left[\begin{array}{cccc}
\gamma_{2} \cos \psi & -\gamma_{4} \sin \psi & 0 & 0 \\
\gamma_{2} \sin \psi & \gamma_{4} \cos \psi & 0 & 0 \\
0 & 0 & \gamma_{6} & 0 \\
0 & 0 & 0 & \gamma_{8}
\end{array}\right] \\
R & =\left[\begin{array}{cccc}
\cos \psi & -\sin \psi & 0 & 0 \\
\sin \psi & \cos \psi & 0 & 0 \\
0 & 0 & 1 & 0 \\
0 & 0 & 0 & 1
\end{array}\right] .
\end{aligned}
$$

\subsection{Error Model}

The main control objective is for the quadrotor to follow a given trajectory. In order to deal with the tracking problem, we rewrite the system in terms of the trajectory error and then design the controller to merely stabilize the error system. Let's define $\mathbf{e}=\zeta-\zeta_{\mathbf{d}}$ as the tracking error and write

$$
\dot{\mathrm{e}}=\left[\begin{array}{c}
\ddot{q}-\ddot{q}_{d} \\
\dot{q}-\dot{q}_{d}
\end{array}\right]=\left[\begin{array}{cc}
-N R^{T} & 0 \\
I & 0
\end{array}\right]\left[\begin{array}{l}
\dot{q} \\
q
\end{array}\right]-\left[\begin{array}{c}
\ddot{q}_{d} \\
q_{d}
\end{array}\right]+\left[\begin{array}{c}
M \\
0
\end{array}\right] u .
$$

Adding and subtracting $N R^{T} \dot{q}_{d}$ in (4), we have

$$
\begin{aligned}
\dot{\mathbf{e}} & =\left[\begin{array}{c}
-N R^{T} \dot{q}-\ddot{q}_{d}+N R^{T}\left(\dot{q}_{d}-\dot{q}_{d}\right) \\
\dot{q}-\dot{q}_{d}
\end{array}\right]+\left[\begin{array}{c}
M \\
0
\end{array}\right] u \\
& =\left[\begin{array}{c}
-N R^{T}\left(\dot{q}-\dot{q}_{d}\right)-\ddot{q}_{d}-N R^{T} \dot{q}_{d} \\
\dot{q}-\dot{q}_{d}
\end{array}\right]+\left[\begin{array}{c}
M \\
0
\end{array}\right] u .
\end{aligned}
$$

Rewriting (5) in the state space form, we have 


$$
\begin{aligned}
\dot{\mathrm{e}} & =\left[\begin{array}{cc}
-N R^{T} & 0 \\
I & 0
\end{array}\right]\left[\begin{array}{c}
\dot{q}-\dot{q}_{d} \\
q-q_{d}
\end{array}\right]+\left[\begin{array}{c}
-\ddot{q}_{d}-N R^{T} \dot{q}_{d}+M u \\
0
\end{array}\right] \\
& =\left[\begin{array}{cc}
-N R^{T} & 0 \\
I & 0
\end{array}\right] \mathbf{e}+\left[\begin{array}{l}
I \\
0
\end{array}\right] \nu
\end{aligned}
$$

where $\nu=-\ddot{q}_{d}-N R^{T} \dot{q}_{d}+M u$. The actual control input to be sent to the system is calculated by

$$
u=M^{-1}\left(\nu+\ddot{q}_{d}+N R^{T} \dot{q}_{d}\right) .
$$

where $\nu$ is given by a state feedback law to be designed to stabilize the error system as shown next.

\section{TAKAGI-SUGENO FUZZY MODELLING AND CONTROLLER}

In this work, it is proposed a fuzzy controller for the nonlinear error system (6). To obtain the fuzzy controller we describe the nonlinear system as a TS fuzzy system. Considering the sector nonlinearity approach, system (6) can be represented as

$$
\dot{\mathbf{e}}(t)=\sum_{i=1}^{4} \sum_{j=1}^{4} h_{i}(\psi) h_{j}(\psi)\left[\begin{array}{cc}
-N_{i} R_{j}^{T} & 0 \\
I & 0
\end{array}\right] \mathbf{e}+\left[\begin{array}{l}
I \\
0
\end{array}\right] \nu
$$

where

$$
\begin{array}{rlrl}
N_{1} & =\left[\begin{array}{rrrr}
-\gamma_{2} & \gamma_{4} & 0 & 0 \\
-\gamma_{2} & -\gamma_{4} & 0 & 0 \\
0 & 0 & \gamma_{6} & 0 \\
0 & 0 & 0 & \gamma_{8}
\end{array}\right], & R_{1}=\left[\begin{array}{rrrr}
-1 & 1 & 0 & 0 \\
-1 & -1 & 0 & 0 \\
0 & 0 & 1 & 0 \\
0 & 0 & 0 & 1
\end{array}\right] \\
N_{2}=\left[\begin{array}{rrrr}
-\gamma_{2} & -\gamma_{4} & 0 & 0 \\
\gamma_{2} & -\gamma_{4} & 0 & 0 \\
0 & 0 & \gamma_{6} & 0 \\
0 & 0 & 0 & \gamma_{8}
\end{array}\right], & R_{2}=\left[\begin{array}{rrrr}
-1 & -1 & 0 & 0 \\
1 & -1 & 0 & 0 \\
0 & 0 & 1 & 0 \\
0 & 0 & 0 & 1
\end{array}\right] \\
N_{3}=\left[\begin{array}{rrrr}
\gamma_{2} & \gamma_{4} & 0 & 0 \\
-\gamma_{2} & \gamma_{4} & 0 & 0 \\
0 & 0 & \gamma_{6} & 0 \\
0 & 0 & 0 & \gamma_{8}
\end{array}\right], & R_{3}=\left[\begin{array}{rrrr}
1 & 1 & 0 & 0 \\
-1 & 1 & 0 & 0 \\
0 & 0 & 1 & 0 \\
0 & 0 & 0 & 1
\end{array}\right] \\
N_{4}=\left[\begin{array}{rrrr}
\gamma_{2} & -\gamma_{4} & 0 & 0 \\
\gamma_{2} & \gamma_{4} & 0 & 0 \\
0 & 0 & \gamma_{6} & 0 \\
0 & 0 & 0 & \gamma_{8}
\end{array}\right], & R_{4}=\left[\begin{array}{rrrr}
1 & -1 & 0 & 0 \\
1 & 1 & 0 & 0 \\
0 & 0 & 1 & 0 \\
0 & 0 & 0 & 1
\end{array}\right]
\end{array}
$$

are the matrices of the local models and $h_{i}(\psi)$ are the membership functions of each local model given by

$$
\begin{array}{ll}
h_{1}(\psi)=w_{1}^{1}(\psi) w_{1}^{2}(\psi), & h_{2}(\psi)=w_{1}^{1}(\psi) w_{0}^{2}(\psi), \\
h_{3}(\psi)=w_{0}^{1}(\psi) w_{1}^{2}(\psi), & h_{4}(\psi)=w_{0}^{1}(\psi) w_{0}^{2}(\psi),
\end{array}
$$

with

$$
\begin{array}{ll}
w_{0}^{1}(\psi)=\frac{\cos (\psi)+1}{2}, & w_{1}^{1}(\psi)=1-w_{0}^{1}(\psi), \\
w_{0}^{2}(\psi)=\frac{\sin (\psi)+1}{2}, & w_{1}^{2}(\psi)=1-w_{0}^{1}(\psi) .
\end{array}
$$

When convenient, the argument of function $h_{k}(\psi)$ will be omitted.

\subsection{State Feedback Design}

For control purposes, consider the PDC procedure presented in Tanaka and Wang (2001). Thus, the fuzzy controller is given by:

$$
\nu=-\sum_{i=1}^{4} h_{i} \mathbf{K}_{i} \mathbf{e}
$$

with $h_{i}$ defined in (9) and $\mathbf{K}_{i} \in \mathbb{R}^{4 \times 8}$ the local state feedback gains. Replacing (10) in (8), the closed loop system becomes

$$
\dot{\mathrm{e}}(t)=\sum_{i=1}^{4} \sum_{j=1}^{4} h_{i} h_{j}\left(\mathbf{A}_{i j}-\mathbf{B K} \mathbf{K}_{i}\right) \mathbf{e}
$$

where $\mathbf{A}_{i j}=\left[\begin{array}{cc}-N_{i} R_{j}^{T} & 0 \\ I & 0\end{array}\right]$ and $\mathbf{B}=\left[\begin{array}{l}I \\ 0\end{array}\right]$. Thus, the control purpose is to find matrices $\mathbf{K}_{i}$, such that system (11) is asymptotically stable.

\subsection{Sufficient Stabilizing LMI Conditions}

The results are developed considering a Lyapunov function candidate $V(\mathbf{e}(t)): S \rightarrow \mathbb{R}$ as follows:

$$
V(\mathbf{e}(t))=\sum_{i=1}^{4} h_{i} \mathbf{e}(t)^{\prime} \mathbf{P}_{i} \mathbf{e}(t)
$$

where $S$ is a subset of $\mathbb{R}^{8}$. As a consequence, the derivative of (12) is given by

$$
\begin{aligned}
\dot{V}(\mathbf{e}(t))=\sum_{i=1}^{4} h_{i}\left(\dot{\mathbf{e}}(t)^{\prime} \mathbf{P}_{i} \mathbf{e}(t)\right. & \left.+\mathbf{e}(t)^{\prime} \mathbf{P}_{i} \dot{\mathbf{e}}(t)\right) \\
& +\sum_{\rho=1}^{4} \dot{h}_{\rho} \mathbf{e}(t)^{\prime} \mathbf{P}_{\rho} \mathbf{e}(t) .
\end{aligned}
$$

The first-order time-derivative of the membership function $h_{i}, i=1, \ldots, 4$, appears in $\dot{V}(\mathbf{e}(t))$. By (9), follows that

$$
\sum_{i=1}^{4} h_{i}=1 \Rightarrow \sum_{i=1}^{4} \dot{h}_{i}=0
$$

Thus, the derivative of $V(\mathbf{e}(t))$ (13) is nonconvex and, to obtain the LMI conditions the following set is defined

$$
\mathcal{D}=\left\{\mathbf{e}(t) \in S:\left|\dot{h}_{\rho}\right| \leq \phi_{\rho}, \quad \forall \rho \in \mathcal{R}\right\}
$$

where $\mathcal{R}=\{1,2,3,4\}$ and $\phi_{\rho}$ are given positive real numbers. The following lemma is used in the main result. Lemma 1. Let $\Psi_{i j}, i, j \in \mathcal{R}$, be matrices of proper dimensions. If LMIs

$$
\begin{aligned}
& \Psi_{i i} \prec \mathbf{0}, \quad \forall i \in \mathcal{R}, \\
& \frac{1}{r-1} \Psi_{i i}+\Psi_{i j}+\Psi_{j i} \prec \mathbf{0}, \quad \forall i, j \in \mathcal{R}, i<j,
\end{aligned}
$$

holds. Then,

$$
\sum_{i=1}^{4} \sum_{j=1}^{4} h_{i} h_{j} \Psi_{i j} \prec \mathbf{0}
$$

Proof. See Tuan et al. (2001).

The main result proposes a sufficient condition for the asymptotic stability of system (11) using function (12). 
Theorem 2. Let $\phi_{\rho}$ be known positive real numbers satisfying (14). If for a positive constant $\mu$, there exist matrices $\mathbf{Z} \in \mathbb{R}^{8 \times 8}, \mathbf{Y}_{i} \in \mathbb{R}^{4 \times 8}, \mathbf{X}=\mathbf{X}^{\prime} \in \mathbb{R}^{8 \times 8}$ and $\mathbf{Q}_{i}=\mathbf{Q}_{i}^{\prime} \in$ $\mathbb{R}^{8 \times 8}$ satisfying (15)-(18), then feedback system (11), with local gains $\mathbf{K}_{i}=\mathbf{Y}_{i} \mathbf{Z}^{-1}$, is asymptotically stable for any $\mathbf{e}(t) \in \mathcal{D}$.

$$
\begin{aligned}
\mathbf{Q}_{i} & \succ \mathbf{0}, \quad \forall i \in \mathcal{R}, \\
\mathbf{Q}_{\rho}+\mathbf{X} \succeq \mathbf{0}, \quad \forall \rho \in \mathcal{R}, & \\
\Psi_{i i} & \prec \mathbf{0}, \quad \forall i \in \mathcal{R}, \\
\frac{1}{r-1} \Psi_{i i}+\Psi_{i j}+\Psi_{j i} & \prec \mathbf{0}, \quad \forall i, j \in \mathcal{R}, i<j
\end{aligned}
$$

where

$$
\begin{aligned}
\Psi_{i j} & =\left[\begin{array}{cc}
\tilde{\mathbf{Q}}-\mathbf{A}_{i j} \mathbf{Z}-\mathbf{Z}^{\prime} \mathbf{A}_{i j}^{\prime}+\mathbf{B} \mathbf{Y}_{i}+\mathbf{Y}_{i}^{\prime} \mathbf{B}^{\prime} & \star \\
\mathbf{Q}_{i}-\mu\left(\mathbf{A}_{i j} \mathbf{Z}-\mathbf{B} \mathbf{Y}_{i}\right)+\mathbf{Z}^{\prime} & \mu\left(\mathbf{Z}+\mathbf{Z}^{\prime}\right)
\end{array}\right] \\
\tilde{\mathbf{Q}} & =\sum_{\rho=1}^{4} \phi_{\rho}\left(\mathbf{Q}_{\rho}+\mathbf{S}\right)
\end{aligned}
$$

Proof. If (17) and (18) hold, by Lemma 1, it yields

$$
\sum_{i=1}^{4} \sum_{j=1}^{4} h_{i} h_{j} \Psi_{i j} \prec \mathbf{0} .
$$

Considering $\mathbf{K}_{i}=\mathbf{Y}_{i} \mathbf{Z}^{-1}$, from Faria et al. (2013), the inequality above ensures that

$$
\begin{array}{r}
{\left[\sum_{i=1}^{4} \sum_{j=1}^{4} h_{i} h_{j}\left(\mathbf{A}_{i j}-\mathbf{B} \mathbf{K}_{i}\right)\right]^{\prime}\left(\sum_{i=1}^{4} h_{i} \mathbf{P}_{i}\right)} \\
+\left(\sum_{i=1}^{4} h_{i} \mathbf{P}_{i}\right)\left[\sum_{i=1}^{4} \sum_{j=1}^{4} h_{i} h_{j}\left(\mathbf{A}_{i j}-\mathbf{B} \mathbf{K}_{i}\right)\right] \\
\sum_{\rho=1}^{4} \phi_{\rho} \mathbf{P}_{\rho} \prec \mathbf{0} .
\end{array}
$$

Thus, premultiplying and posmultiplying inequality (19) by $\mathbf{e}(t)^{\prime}$ and its transpose, respectively. By (11), (12) and (13), it follows that

$$
\dot{V}(\mathbf{e}(t))<\mathbf{0}
$$

Moreover, by (15), $V(\mathbf{e}(t))>\mathbf{0} \forall \mathbf{e}(t) \neq \mathbf{0}$. Therefore, system (11) is asymptotically stable for any $\mathbf{e}(t) \in \mathcal{D}$.

\section{CONTROL IMPLEMENTATION}

The experiments were carried out with the commercially available Parrot BEBOP 2.0 (see Figure 1). The control system described in Section 2 was implemented using the Robot Operating System (ROS) (Quigley et al., 2009) and its bebop_autonomy, drone_dev and vicon_bridge packages. The bebop_autonomy driver allows data exchange with the quadrotor to send motion and configuration commands and read on-board sensors such as the IMU and GPS. The package drone_dev is used for implementing control systems on off-the-shelf quadrotors (Benevides et al., 2019). It builds on bebop_autonomy's tools, having the control system structure implemented. Finally, we have the vicon_bridge driver which provides data from VICON motion capture systems. The vicon_bridge node connects to the tracking software via the DataStream API and publishes the information as geometry_msgs/TransformStamped messages in the vicon/bebop/bebop topic.

\subsection{Trajectory Generation}

A ROS node was created to publish the desired position to the /bebop/waypoint topic where the controller is subscribed. This node runs at $50 \mathrm{~Hz}$ and publishes messages of type nav_msgs/Odometry containing the following information

$$
q_{d}(t)=\left[\begin{array}{l}
x_{d}(t) \\
y_{d}(t) \\
z_{d}(t) \\
\psi_{d}(t)
\end{array}\right]=\left[\begin{array}{c}
a \cos \omega t \\
a \sin \omega t \\
0 \\
0
\end{array}\right]
$$

where $a$ is the radius and $\omega=\frac{v}{a}$ is the angular velocity.

\subsection{State Measurement and Estimation}

The VICON motion capture system tracks the position and orientation of the quadrotor in the data source software. The vicon_bridge node makes this information available by publishing it. However, the orientation is given in quaternions and must be converted to Euler angles before useing it in the computation of the control law.

To build the full tracking error state, we estimate the quadrotor velocity using a Kalman filter. For this, we used a kinematic constant velocity model given by

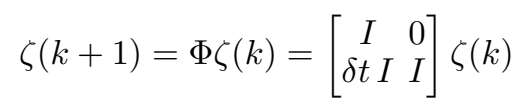

where $\zeta$ is the state vector defined in Section $3, \Phi$ is the state transition matrix and $\delta t$ is the time interval it takes to get an update of the position measurement. The observation model is given by

$$
m(k)=H \zeta(k)=\left[\begin{array}{ll}
0 & I
\end{array}\right] \zeta(k) .
$$

The equations for the filter are given by

$$
\begin{array}{r}
\zeta_{p}(k+1)=\Phi \zeta_{e}(k) \\
P_{p}=\Phi P \Phi^{T}+Q \\
K=P_{p} H^{T}\left(H P_{p} H^{T}+R\right)^{-1} \\
\zeta_{e}=\zeta_{p}+K\left(z-H \zeta_{p}\right) \\
P=P_{p}-K H P_{p}
\end{array}
$$

where $\zeta_{e}$ is the filter state estimation, $P$ is the error covariance matrix, $K$ is the Kalman gain, $Q$ is the process noise covariance matrix, and $R$ is the measurement noise covariance matrix.

\subsection{Fuzzy Controller}

The PDC fuzzy controller was implemented as a ROS node, generating the following control signal

$$
u=M^{-1}\left(-\sum_{i=1}^{4} h_{i} K_{i} e+\ddot{q}_{d}+N R^{T} \dot{q}_{d}\right)
$$


where $M$ and $N R^{T}$ are the model matrices, $h_{i}, i=1, \ldots, 4$, are the fuzzy membership functions, $e$ is the tracking error signal and $\dot{q}_{d}$ and $\ddot{q}_{d}$ indicate the desired velocity and acceleration, respectively. The signal is published to cmd_vel topic as geometry_msgs/Twist messages

\subsection{Node Communication}

The configuration of the communication among the various devices of the control system is shown in Fig. 3. The host PC runs basically 5 ROS nodes:

- drone_driver: responsible for sending commands to the quadrotor. It is part of the bebop_autonomy package.

- vicon bridge: responsible for measuring the quadrotor's position and orientation. It is part of the vicon_bridge package.

- traj_planner: responsible for sending the actual controller desired position, velocity and acceleration according to a given trajectory. It is part of the drone_dev package.

- drone_dev: controller node. Responsible for calculating the control signal. It is part of the drone_dev package.

- bebop_joy: responsible for sending joystick commands to the quadrotor. In this implementation, it is used as a security measure (dead man's switch). It is part of the bebop_autonomy package.

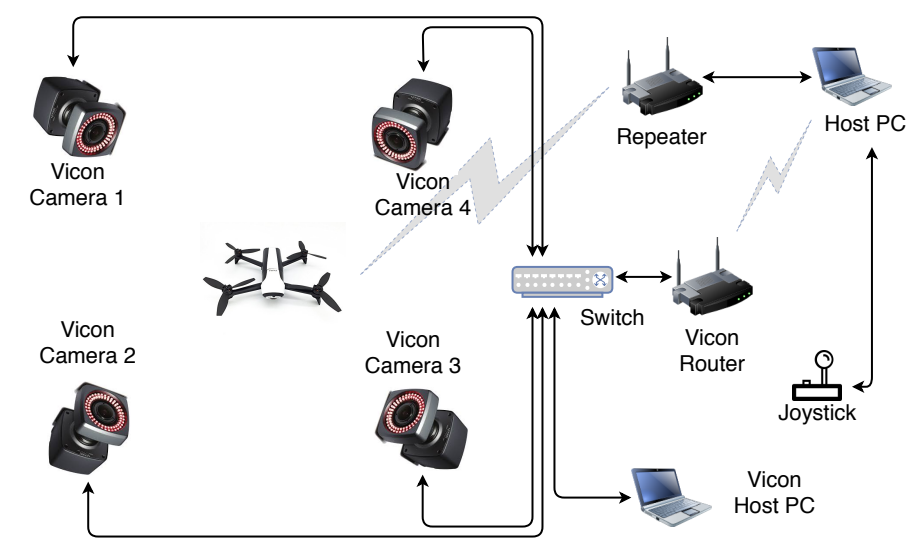

Figure 3. Control system components (Benevides et al., 2019).

\section{RESULTS}

The trajectory parameters we chose are $a=0.3 \mathrm{~m}$ and $v=$ $0.1 \mathrm{~m} / \mathrm{s}$, which give an angular velocity of $\omega=3.33 \mathrm{rad} / \mathrm{s}$. The model parameters $\gamma_{i}, i=1, \cdots, 8$ were previously identified via a least squares method and are given by

$$
\left[\begin{array}{c}
\gamma_{1} \\
\vdots \\
\gamma_{8}
\end{array}\right]=\left[\begin{array}{c}
4.3321 \\
0.2333 \\
4.1220 \\
0.4145 \\
4.4206 \\
3.1341 \\
5.9289 \\
-0.3868
\end{array}\right]
$$

The project parameters for the LMI conditions are $\mu=0.1$ and $\phi_{\rho}=0.1 \forall \rho$. The controller gains are given in (30).

Figure 4 shows the $x$ coordinate of the quadrotor pose as a function of time. Initially, the error is large, but the controller is able to take the system to the reference trajectory. We can notice the small amplitude error in the sinusoidal peak. Figure 5 shows the $y$ coordinate of the quadrotor pose as a function of time. Here the amplitude error is larger. We can decrease these errors by improving the model used for the control design and adding uncertainties.

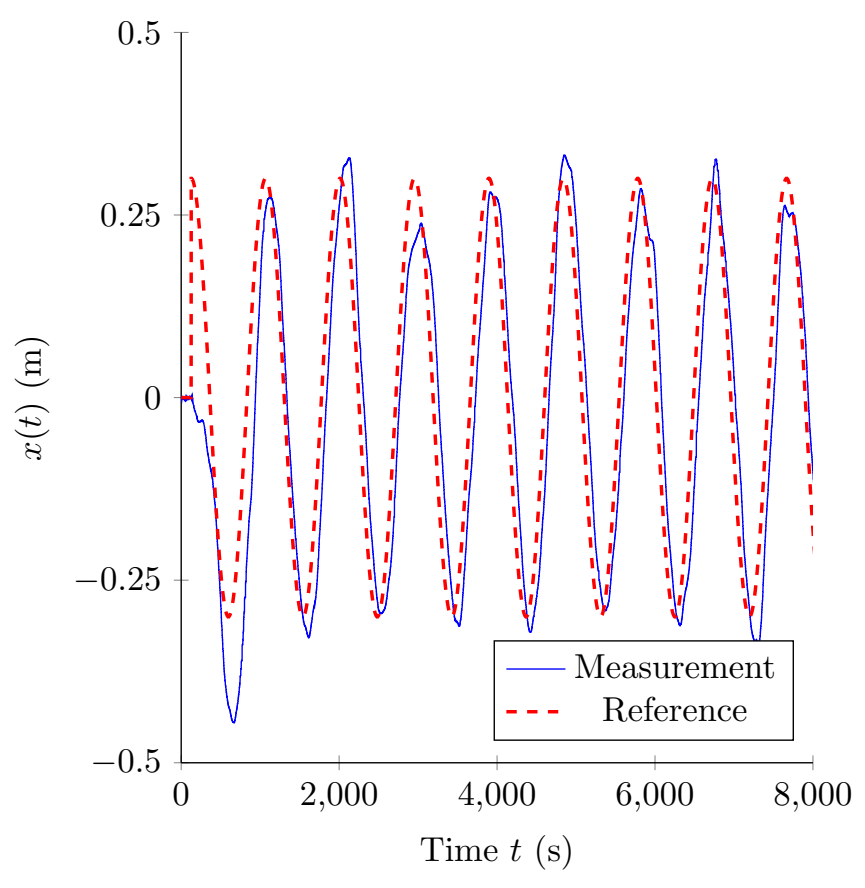

Figure 4. Position $x(t)$

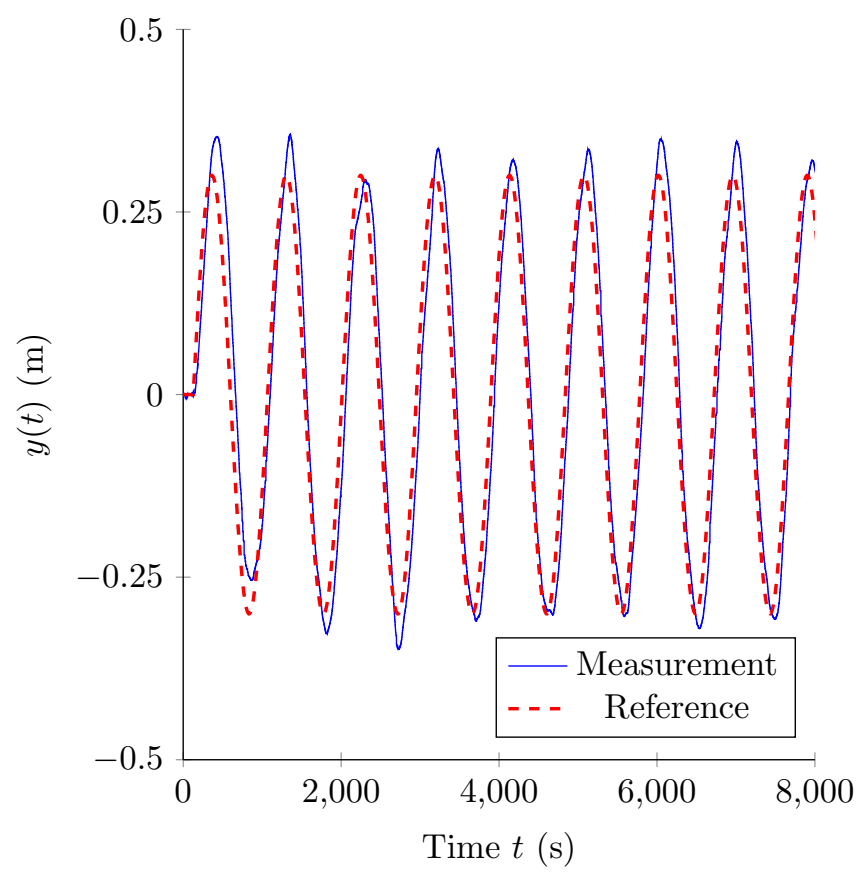

Figure 5. Position $y(t)$ 


$$
\begin{aligned}
K_{1}= & {\left[\begin{array}{cccccccc}
1.0338 & 0.1834 & 0 & 0 & 0.4511 & 0.0002 & 0 & 0 \\
0.1834 & 1.0338 & 0 & 0 & 0.0002 & 0.4511 & 0 & 0 \\
0 & 0 & -2.6293 & 0 & 0 & 0 & 1.0225 & 0 \\
0 & 0 & 0 & 0.8916 & 0 & 0 & 0 & 1.0225
\end{array}\right] } \\
K_{2}= & {\left[\begin{array}{cccccccc}
1.0338 & 0.1834 & 0 & 0 & 0.4511 & 0.0002 & 0 & 0 \\
0.1834 & 1.0338 & 0 & 0 & 0.0002 & 0.4511 & 0 & 0 \\
0 & 0 & -2.6293 & 0 & 0 & 0 & 1.0225 & 0 \\
0 & 0 & 0 & 0.8916 & 0 & 0 & 0 & 1.0225
\end{array}\right] } \\
K_{3}= & {\left[\begin{array}{cccccccc}
1.0338 & 0.1834 & 0 & 0 & 0.4511 & 0.0002 & 0 & 0 \\
0.1834 & 1.0338 & 0 & 0 & 0.0002 & 0.4511 & 0 & 0 \\
0 & 0 & -2.6293 & 0 & 0 & 0 & 1.0225 & 0 \\
0 & 0 & 0 & 0.8916 & 0 & 0 & 0 & 1.0225
\end{array}\right] } \\
K_{4}= & {\left[\begin{array}{cccccccc}
1.0338 & 0.1834 & 0 & 0 & 0.4511 & 0.0002 & 0 & 0 \\
0.1834 & 1.0338 & 0 & 0 & 0.0002 & 0.4511 & 0 & 0 \\
0 & 0 & -2.6293 & 0 & 0 & 0 & 1.0225 & 0 \\
0 & 0 & 0 & 0.8916 & 0 & 0 & 0 & 1.0225
\end{array}\right] }
\end{aligned}
$$

\section{CONCLUDING REMARKS}

We implemented a ROS-based control system with a PDC fuzzy controller. The platform used was a BEBOP Parrot 2.0 and the control objective was to follow a given trajectory (a circle). Experimental results showed overall success in accomplishing this task. A set back in using an off-the-shelf platform is the lack of access to the inner control loop. Future works will consider model uncertainty and convergence rates or another performance index in the LMIs.

\section{REFERENCES}

Benevides, J.R.S., Inoue, R.S., Paiva, M.A.D., and Terra, M.H. (2019). ROS-based robust and recursive optimal control of commercial quadrotors. In 15th International Conference on Automation Science and Engineering (CASE). Accepted.

Bresciani, T. (2008). Modelling, Identification and Control of a Quadrotor Helicopter. Master thesis, Department of Automatic Control, Lund University.

Faria, F.A., Silva, G.N., and Oliveira, V.A. (2013). Reducing the conservatism of LMI-based stabilization conditions for TS fuzzy systems using fuzzy Lyapunov functions. International Journal of Systems Science, 44(10), 1956-1969.

Grijalva, J.G., Klug, M., and Castelan, E.B. (2017). Control of a Quadrotor Under Sensors Sampling Limitations Using TS Fuzzy Modeling: A Discrete Time Approach. In Simpósio Brasileiro de Automacao Inteligente. Porto Alegre, RS.

Koslosky, E., de Oliveira, A.S., Wehrmeister, M.A., and Fabro, J.A. (2017). Designing Fuzzy Logic Controllers for ROS-Based Multirotors. In Studies in Computational Intelligence, volume 707, 41-82. Springer Verlag.
Mo, H. and Farid, G. (2019). Nonlinear and adaptive intelligent control techniques for quadrotor UAV - a survey. Asian Journal of Control, 21(2), 989-1008.

Ponce, P., Molina, A., Cayetano, I., Gallardo, J., Salcedo, H., and Rodriguez, J. (2015). Experimental fuzzy logic controller type 2 for a quadrotor optimized by ANFIS. In 15th IFAC Symposium on Information Control Problems in Manufacturing, 2435 - 2441. Ontario, Canada.

Quigley, M., Gerkey, B., Conley, K., Faust, J., Foote, T., Leibs, J., Berger, E., Wheeler, R., and Ng, A. (2009). ROS: an open-source robot operating system. In Open-Source Software workshop of the International Conference on Robotics and Automation (ICRA). Kobe, Japan.

Sampath, B.G., Perera, K.C.R., Wijesuriya, W.A.S.I., and Dassanayake, V.P.C. (2014). Fuzzy Based Stabilizer Control System for Quad-Rotor. International Journal of Mechanical, Aerospace, Industrial and Mechatronics Engineering, 8(2), 206-212.

Santana, L.V., Brandao, A.S., Sarcinelli-Filho, M., and Carelli, R. (2014). A trajectory tracking and 3d positioning controller for the AR.Drone quadrotor. In 2014 International Conference on Unmanned Aircraft Systems (ICUAS), 756-767. IEEE.

Tanaka, K. and Wang, H.O. (2001). Fuzzy Control Systems Design and Analysis. John Wiley \& Sons, Inc., New York, USA.

Tang, Y. and Li, Y. (2015). Dynamic modeling for highperformance controller design of a UAV quadrotor. In 2015 IEEE International Conference on Information and Automation, 3112-3117.

Tuan, H.D., Apkarian, P., Narikiyo, T., and Yamamoto, Y. (2001). Parameterized linear matrix inequality techniques in fuzzy control system design. IEEE Transactions on Fuzzy Systems, 9(2), 324-332. 